\title{
Shear capacity prediction of slender reinforced concrete structures with steel fibers using machine learning
}

\author{
Oladimeji Benedict Olalusi ${ }^{\text {a, , }}$ Paul O. Awoyera ${ }^{\text {b,c,d }}$ \\ ${ }^{a}$ Department of Civil Engineering, Structural Engineering \& Computational Mechanics Group (SECM), University of KwaZulu-Natal, Durban, South Africa \\ ${ }^{\mathrm{b}}$ Department of Civil Engineering, Covenant University, Ota, Nigeria \\ ${ }^{c}$ Institute of Research and Development, Duy Tan University, Da Nang 550000, Vietnam \\ ${ }^{\mathrm{d}}$ Faculty of Civil Engineering, Duy Tan University, Da Nang 550000, Vietnam
}

\section{A R T I C L E I N F O}

\section{Keywords:}

Steel fiber reinforced concrete beam

Shear capacity

Structural design

Model uncertainty

Partial safety factors

Structural reliability

Artificial intelligence

\begin{abstract}
A B S T R A C T
Shear failure in reinforced concrete beams poses a critical safety issue since it may occur without any prior signs of damage in some cases. Many of the existing shear design equations for steel fiber reinforced concrete (SFRC) beams include significant uncertainty due to failure in reflecting the phenomenology of shear resistance accurately. Given these, adequate reliability evaluation of shear design provisions for SFRC beam is of high significance, and increased accuracy and minimisation of variability in the predictive model is essential. This contribution proposes machine learning (ML) based methods - Gaussian Process regression (GPR) and the Random Forest (RF) techniques - to predict the ultimate shear resistance of SFRC slender beams without stirrups. The models were developed using a database of 326 experimental SFRC slender beams obtained from previous studies, utilising $75 \%$ for model training and the remainder for testing. The performance of the proposed models was assessed by statistical comparison to experimental results and to that of the state-of-practice existing shear design models (fib Model Code 2010, German guideline, Bernat et al. model). The proposed ML-based models are in close alignment with the experimentally observed shear strength and the existing predictive models, but provide more accurate and unbiased predictions. Furthermore, the model uncertainty of the various resistance models was characterised and investigated. The ML-based models displayed the lowest bias and variability, with no significant trend with input parameters. The inconsistencies observed in the predictions by the existing shear design formulations at the variation of shear span to effective depth ratio is a major cause for concern; reliability analysis is required. Finally, partial resistance safety factors were proposed for the model uncertainty associated with the existing shear design equations.
\end{abstract}

\section{Introduction}

Given the quasi-brittle behaviour of concrete in tension, shear failure in reinforced concrete (RC) beams without web reinforcements (stirrups) can lead to disastrous consequences. Shear failure, which is often initiated by inclined cracks, is an undesirable mode of failure which can occur without any prior warning. The different modes of shear failure in RC beams without stirrups include diagonal-tension, shear-compression and web-crushing $[1,2]$. The concept of incorporating fiber reinforcements to improve the behaviour of concrete has been introduced since ancient time $[3,4]$. Fibers are produced from various types of materials, namely steel, carbon, plastic, glass and natural fibers (coconut (coir) fiber, bamboo fiber, wood fiber, sisal fiber) $[5,6]$. Steel fibers are the most prevalent form of fibers used in reinforced concrete members. This can be attributed to their capability to restrain crack opening, enhance ductile and post-cracking behaviour; increase flexural toughness, tensile strength, energy absorption and improve shear behaviour [7-10]. Over the past decades, extensive experimental and numerical studies have been undertaken on the shear strength of SFRC beams [914]. The various studies reported that the inclusion of steel fibers in concrete structural members greatly improves their shear capacity and ductility.

The shear behaviour of SFRC beam is influenced by several parameters and mechanism like the conventional reinforced concrete beams. There is no yet an international consensus on some of the critical parameters and mechanisms governing the shear behaviour of SFRC beams

\footnotetext{
* Corresponding authors.

E-mail addresses: olalusio@ukzn.ac.za, dimejibenlusy@yahoo.co.uk (O.B. Olalusi).
} 
[15]. Some of these parameters include the effective member depth $d$, the concrete compressive strength $f_{c}$, the aggregate size $d_{a g}$, the shearspan to effective depth $a / d$, and amount of longitudinal reinforcement ratio $\rho$, the volume percentage of fibers, aspect ratio (ratio of length to the diameter of fibers) and shape of steel fibers [16]. Reinforced concrete beams can be categorised on the basis of the shear span to effective depth $a / d$ ratio. Slender beams are those with a/d $>2.5$ and may fail either in shear or in flexure $[1,17]$.

Shear failure poses a crucial safety issue since it may develop swiftly with no prior signs of damage. Consequently, shear design provisions should be reliable and accurate. Numerous design expressions have been introduced in the current design standards [18-20] and literature. However, developing a precise and acceptable design formulation for shear capacity of SFRC beams is still an evolving process. A summary of most of the proposed shear design models for SFRC beams without stirrups in current literature and design guidelines can be found in $[15,16]$. Majority of the existing shear design formulations are either empirical or semi-empirical based, except for the models based on the Modified Compression Field Theory (MCFT) [21,22], the Dual Potential Capacity Model [23], plasticity-based models [24,25] and the modified Multi-Action Shear Model [26]. The comparison of the various SFRC shear resistance models, as presented in $[15,26]$, indicate the difficulty and complexity of the accurate prediction of shear capacity of SFRC beams. Not only do the resistance predictions of the various procedures differ from each other, but they also vary from the experimentally observed shear strength. Given these uncertainties, there is an interest in the adequate assessment of the reliability of SFRC beams in the case of shear failure. To that end, maximum possible accuracy and minimum possible variability in the predictive model are essential. These qualities can also be translated to model uncertainty, which is a quantifiable measure in structural reliability assessments.

In recent years, innovations in computing have led to a boom in the utilisation of artificial intelligence (AI) across all industries. Machine learning (ML) or soft computing (SC) techniques have been extensively applied to a wide range of civil engineering problems such as design optimisation, stochastic simulations, reliability analysis, performance evaluating of structural systems of high complexity [27-32]. Various machine learning approaches such as Random Forest, Gene Expression Programming (GEP), Bayesian Networks, Generic Algorithms, Gaussian Process regression, Support Vector Machines, Artificial Neural Networks (ANNs), have been successfully implemented in modelling different structural engineering problems [32-37]. However, a survey of available literature indicates that previous studies on ML-based modelling of shear strength of SFRC beams are limited. Most of the available studies adopt popular tools such as ANN [37-41] and GEP [42-49] for modelling the shear resistance of SFRC beams using an experimental database containing both slender and non-slender beams. Previous studies [50,51] identified some of the major challenges facing the successful implementation of ANN as the problem of over-fitting, problem of obtaining the optimum hyperparameters and suitable network topology, poor generalising performance and slow convergence speed.

Even though ANN and GEP algorithms have been well applied in several studies involving the shear capacity prediction of SFRC beams, more efficient and accurate machine learning algorithms should be investigated for more extensive database and recent experimental results. More recent studies present soft computing techniques such as the Gaussian Process regression (GPR) and the Random Forest (RF) with high efficiency in modelling complex problems in structural engineering [32,51-55]. Both algorithms have been successfully implemented to solve nonlinear, small samples and high-dimensional issues [32,51-62]. Some of the advantages of Gaussian Process includes meaningful hyperparameters, its good generalisation capability, no required predefined structure, the tendency to incorporate prior knowledge and specifications via the selection of kernel functions and, directly captures the model uncertainty [51]. Besides GPR and RF, other ML techniques including, Bayesian networks, or hybrid methods such as adaptive neuro-fuzzy inference systems, have been investigated as possible candidates for efficient strength modelling.

The main aim of this contribution is to propose efficient ML-based shear resistance models for SFRC slender beams without stirrups and to characterise the model uncertainties in shear resistance models. To this aim, two machine learning algorithms are adopted, namely the Gaussian Process regression (GPR) and the Random Forest techniques. These ML-based algorithms are applied for the first time in predicting the shear strength of SFRC slender beams. At first, an overview of some of the existing shear predictive models for SFRC beams without stirrups are briefly presented (Section 2). The background of the proposed machine learning techniques is discussed in Section 3. Using an experimental database of 326 specimens on SFRC slender beam tests, from the database compiled by [15], the models are trained and tested. The experimental database is discussed in Section 4. A comparative assessment of the developed ML-based models with experimentally observed shear strength is conducted and discussed. The developed ML-based models are assessed as regards their accuracy and efficiency compared to the state-of-practice predictive model embedded in international design standards and literature (Section 5). An estimate of resistance partial safety factors for model uncertainties associated to shear resistance models for SFRC beams is proposed in Section 5. While other MLbased algorithms (such as ANN and GEP) have been mostly applied in predicting the shear resistance of SFRC beams in the literature studies mentioned earlier, this contribution is thus a pioneer work in the application of the Gaussian Process regression and Random Forest techniques to model the shear resistance of SFRC beams, and also an improvement of the state-of-the-art. The outcome of this study is valuable for the establishment of reliability-based design codes for shear resistance of SFRC beams.

\section{Predictive equations for the shear resistance of SFRC beams without stirrups}

This section presents a summary of the current shear design models for SFRC beams without stirrups from design guidelines and recently published literature. The presented shear strength formulations are later considered in Section 4 for comparative study.

\subsection{German guideline (DAfStB) [19]}

The mean shear resistance of SFRC beam is given as a summation of shear capacity of the concrete contribution and fiber contribution expressed as Eq. (1).

$V_{D A f S t B}=0.15 k b_{w} d\left(100 \rho_{l} f_{c m}\right)^{1 / 3}+\alpha_{c}^{f} f_{c t, R u}^{f} b_{w} h$

$f_{c t, R u}^{f}=k_{F}^{f} k_{G}^{f} 0.37 f_{c f, L 2}^{f}$

To obtain the values of $f_{c f l, L 2}^{f}$, the expressions in Eq. (3) is used [62]. Also, applied in the assessment conducted in [15]

$f_{\text {spfc }}=0.63 \sqrt{f_{\text {cuf }}}+0.288 F \sqrt{f_{\text {cuf }}}+0.052 F$

where $b_{w}$ and $d$ is the width and effective depth of beam crosssection, respectively.

$k$ is the size effect factork $=1+\left(\frac{200}{d}\right)^{0.5} \leq 2$

$f_{c m}$ is the mean concrete strength

$f_{c t, R u}^{f}$ is the tensile strength of SFRC

$\rho_{l}$ is the reinforcement ratio

$k_{F}^{f}$ is fiber orientation factor taken as 0.5 for shear

$f_{c f l, L 2}^{f}$ is the post-cracking flexural strength for a deflection of $3.5 \mathrm{~mm}$

$A_{c t}^{f}$ is the effective area expressed as $A_{c t}^{f}=b_{w} * \min (d, 1.5 \mathrm{~m})$

$k_{G}^{f}$ is the fiber size factor expressed as $k_{G}^{f}=1.0+0.5 A_{c t}^{f} \leq 1.7$ 
$\alpha_{c}^{f}$ is the factor accounting for the long term effects

$F$ is the fiber factor

$h$ is the height of cross-section.

\subsection{Model Code 2010 [20]}

The mean shear resistance of SFRC beams without stirrups is expressed as Eq. (4).

$V_{M C-10}=0.18 k b_{w} d\left[100 \rho_{l} \cdot f_{c m}\left(1+7.5 \cdot \frac{f_{F t u}}{f_{c t}}\right)\right]^{1 / 3}$

Since the information on the tensile strength is not reported in the database utilised in the comparative assessment conducted in Section 4, $f_{F t u}$ is estimated similarly as the German guideline in this study.

where $f_{c t}$ is the tensile strength of concrete without fiber. $f_{F t u}$ is the ultimate tensile strength for the fiber reinforced concrete.

\subsection{Modified multi-action shear model for SFRC beams [26]}

The proposed modified MASM formulation for estimating the shear strength of SFRC beam without stirrups is expressed as Eq. (5).

$V_{M-M A S M}=\left[\zeta\left(0.84-0.10 \frac{\sigma_{t u}}{f_{c t m}}\right) \frac{x}{d}+0.08+1.10 \frac{\sigma_{t u}}{f_{c t m}}\right] f_{c t m} b_{w} d$

The average residual tensile stress $\sigma_{t u}$ is obtained using Eq. (6)

$\frac{\sigma_{t u}}{f_{c t}}=2 \eta_{0} \eta_{1} F$

where $x$ is the neutral axis of plain concrete.

$\zeta$ is the size and slenderness effect factor.

$\eta_{0}$ is the fiber orientation factor taken as 0.405

$\eta_{1}$ is the length efficiency factor for fiber

\section{Machine learning algorithms and performance evaluation}

\subsection{Gaussian Process (GP)}

The Gaussian Process is a powerful tool that provides a probabilistic, non-parametric supervised learning technique for classification and regression problems. Supervised learning is concerned with inferring the values of response variables (or outputs), for a given set of inputs or predictor variables. This study is focused only on the regression aspect. The GP method has received extensive attention due to its capability and effectiveness to handle nonlinear data using kernel machine functions. This technique is presently well accepted and utilised in different fields of structural engineering [51-57]. The GP regression is summarised in this section; further description can be found in [63]. A Gaussian distribution is a continuous probability distribution, with a mean vector $\mu$ and covariance: $\boldsymbol{x}^{\sim} N(\mu, \sigma)$ [63]. A univariate Gaussian distribution, which has a mean vector and covariance can be defined by the function expressed in Eq. (7)

$f(x)=\frac{1}{\sqrt{2 \pi \sigma^{2}}} e^{-\frac{(x-\mu)^{2}}{2 \sigma^{2}}}$

The Gaussian Process is a Gaussian random function having a covariance function $k\left(x, x^{\prime}\right)$ and a mean function $m(x)$, expressed in Eq. (8). The equation represents that the function $f$ is distributed as a GP with mean function $m$ and covariance function $k$.

$f(x)^{\sim} G P\left(m(x), k\left(x, x^{\prime}\right)\right)$

The covariance function/kernel function, $k\left(x, x^{\prime}\right)$, can be found in any function that uses any two arguments, such that $k\left(x, x^{\prime}\right)$, generates a nonnegative definitive covariance matrix $K$. GP has different types of possible covariance functions, or kernel functions some of which includes squared exponential kernel (Eq. (9)), exponential kernel (Eq.
(10)), Matern 5/2 (Eq. (11)) and rational quadratic kernel (Eq. (12)). This study employs the four kernel functions to predict the shear capacity of SFRC slender beams.

$k\left(x, x^{\prime}\right)=\sigma_{f}^{2} \exp \left(-\frac{1}{2 l^{2}}\left(x-x^{\prime}\right)^{T}\left(x-x^{\prime}\right)\right)$

$k\left(x, x^{\prime}\right)=\sigma_{f}^{2} \exp \left(\frac{-r}{l}\right)$

$k\left(x, x^{\prime}\right)=\sigma_{f}^{2}\left(1+\frac{\sqrt{5 r}}{l}+\frac{5 r^{2}}{3 l^{2}}\right) \exp \left(-\frac{\sqrt{5 r}}{l}\right)$

$k\left(x, x^{\prime}\right)=\sigma_{f}^{2}\left(1+\frac{r^{2}}{2 \alpha l^{2}}\right)^{-\alpha}$

where $l$ denotes the length parameter of the kernel function. $r=$ $\sqrt{\left(x-x^{\prime}\right)^{T}\left(x-x^{\prime}\right)} \cdot \alpha$ is a positive-valued scale-mixture parameter. $\sigma_{f}$ and $l$ are hyperparameters

Given we have a set of training data or observations $y$, on which each element is a sample from a Gaussian distribution, representing the real value of the observation affected by some independent Gaussian noise $\varepsilon$ with variance $\sigma_{n}$. The observations can be viewed as being the sum of a function plus an additive Gaussian noise, expressed in Eq. (13) [63].

$y=f(x)+\varepsilon$

Considering this, we can predict a new dependent variable $y_{*}$ given a new test input $x_{*}$. The GP is a set of random variables with a Gaussian distribution having a zero mean. The problem can then be represented as:

$$
\left[\begin{array}{c}
y \\
y_{*}
\end{array}\right] \sim N\left(0,\left[\begin{array}{cc}
K(X, X)+\sigma_{n}^{2} I & K\left(X, X_{*}\right) \\
K\left(X_{*}, X\right) & K\left(X_{*}, X_{*}\right)
\end{array}\right]\right)
$$

The different $K$ matrices are built using any function $k\left(x, x^{\prime}\right)$ able to perform as a covariance function. An elaborate description of the Gaussian Process methodology can be found in $[55,63]$.

\subsection{Random Forest algorithm}

Random forest (or random decision forests, randomised trees) is an ensemble machine learning technique for performing both regression and classification tasks, using multiple decision trees (DT). A random forest $R$ is an ensemble of $T$ independent decisions trees $R=\left\{R_{1}, \cdots, R_{t}\right.$, $\left.\cdots R_{T}\right\}$. In order to construct independent decision trees based on a unique training set, Breiman [64] introduced the randomisation technique known as bagging (also called Bootstrap aggregation). For a given training set $Z=\left\{X^{(n)}, Y^{(n)}\right\}_{n=1}^{N}$, a bootstrap is essentially a subset $Z_{t}$ of the complete training set, in which component has been sampled at random using a uniform distribution, and this, with or without replacement. Each decision tree $R_{t}$ of the ensemble is trained using a different bootstrap $Z_{t}$. The results of the ensemble are obtained by aggregating together, using averaging, the predictions from all individual trees.

Considering the random forest $R=\left\{R_{t}\right\}_{t=1}^{T}$, with each tree $R_{t}$ yielding aa partition $p_{t}$ of the feature space $\chi$. As each individual tree can be seen as a surjective function associating an observation $X \in \chi$ to a cell $A_{t}^{(z t)}$ of partition $p_{t}$, the whole forest is a function which associates $X$ to an ensemble of cells of trees (Eq. (14))

$R(X)=\left\{A_{t}^{(z t)}, \cdots, A_{t}^{(z t)}, \cdots, A_{t}^{(z t)}\right\}$

Considering that each $p_{t}$ is equiprobable, the forest prediction can then be simply computed by averaging the tree posteriors (Eq. (15))

$P(Y \mid X)=\frac{1}{T} \sum_{t=1}^{T} P\left(Y \mid X \in A_{t}^{(z t)}, p_{t}\right)$ 
Breiman [64] demonstrated that the random forest algorithm provides good generalisation capability. Random forests offer a flexible framework with room for selecting task-specific objective functions, different classes of splitting functions or posterior models. The main hyperparameters in Random Forests are the number of trees and the tree depth. Increasing the number of trees makes it possible to average out noisy predictions, and thus corresponds in a monotonic decrease of the prediction error. The maximal allowable depth of the tree is a key parameter that needs to be optimised as it directly impacts the generalisation ability of each tree [65]. The structure of RF is shown in Fig. 1. An elaborate description of the Random Forest methodology can be found in [64].

\section{Construction of the ML-based shear resistance predictive models and performance evaluation}

A brief discussion of the various stages involved the development of the ML shear strength predictive models are presented in this section.

\subsection{Processing of the experimental database}

Several experimental investigations have been conducted on the shear strength of SFRC beams without shear stirrups. A large database of 488 experimental on SFRC beams without stirrups was recently compiled by [15]. From the originally collected 488 experiments, a total of 162 beams with the shear-flexure mode of failure and shear-span to effective depth ratio $a / d<2.5$ (non-slender beams) were filtered out, resulting in a subset of 326 experimental tests investigated in this study. The resulting database of 326 experiments was adopted for model development. Specimens in the evaluation database consist of slender beams with a flanged and rectangular cross-section.

All the beam specimens in the database failed majorly by shearcompression and diagonal tension and had an $a / d$ ratio greater or equal to 2.5. The experimental database covers a wide variety of shear configurations from small to large geometry and low to high reinforcement. The statistical properties of the main parameters of the evaluation database are presented in Table 1 . The main parameters include the shear capacity $V_{u}$, the ratio of the shear span to effective depth $a / d$, beam effective depth $d$, beam width $b_{w}$, amount of longitudinal reinforcement $\rho_{l}$, concrete strength $f_{c}$, aggregate size $d_{a}$, the steel fiber factor $F_{s f}$. The steel fiber factor is a function of the percentage volume $V_{f}$, diameter $d_{f}$ and length of fibers $L_{f}$ (see Eq. (16)).

$F_{s f}=\frac{V_{f} L_{f}}{d_{f}}$

The shear capacity $V_{u}$ of the SFRC beams as a function of the main shear parameters are presented in Fig. 2. Generally, as shown in Table 1 and Fig. 2, the range of parameters of the database corresponds to what can be found practical design situations. Although data for large beam sizes are few, the dataset is deemed representative of most real applications; and that of design situations covered by current design provisions.

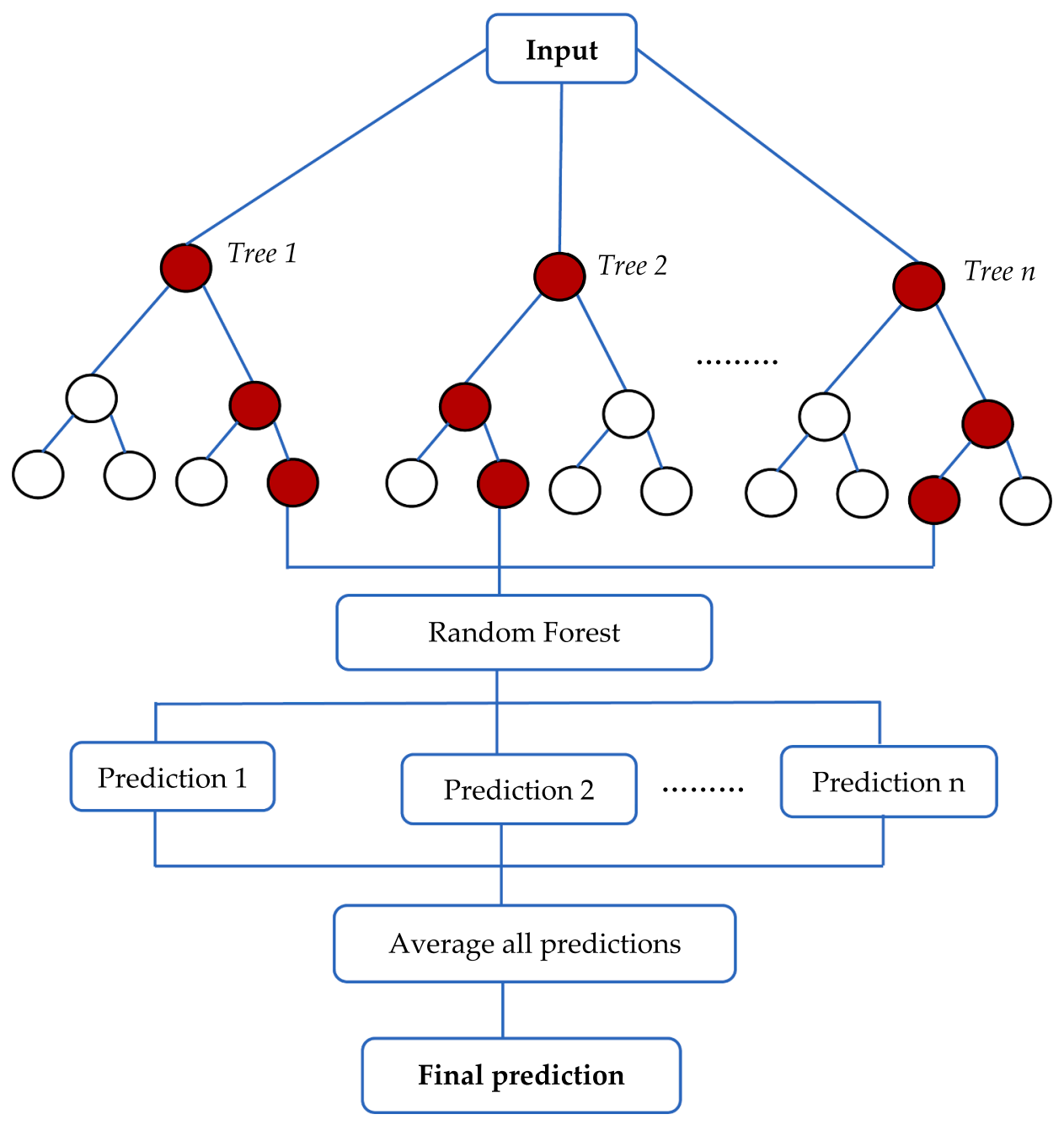

Fig. 1. Schematic diagram for random forest prediction. 
Table 1

Statistical summary of the experimental evaluation database (326).

\begin{tabular}{|c|c|c|c|c|c|c|c|c|c|}
\hline Parameters & Range & Min & 25th Percentile & 50th Percentile & 75th Percentile & Max & Mean & S.D & C.o.V \\
\hline$V_{u}(\mathrm{kN})$ & 686.64 & 12.89 & 45.84 & 107.38 & 191.33 & 699.53 & 134.44 & 128.75 & 0.96 \\
\hline$F_{s f}(-)$ & 1.90 & 0.10 & 0.30 & 0.50 & 0.64 & 2.00 & 0.51 & 0.31 & 0.62 \\
\hline$f_{t}(\mathrm{MPa})$ & 4653.00 & 260.00 & 1100.00 & 1100.00 & 1200.00 & 4913.00 & 1264.36 & 476.60 & 0.38 \\
\hline$d_{a}(\mathrm{~mm})$ & 21.60 & 0.40 & 9.55 & 10.00 & 12.50 & 22.00 & 9.94 & 5.23 & 0.53 \\
\hline$\rho_{l}$ & 0.05 & 0.00 & 0.02 & 0.03 & 0.03 & 0.06 & 0.03 & 0.01 & 0.38 \\
\hline$f_{c}(\mathrm{MPa})$ & 205.23 & 9.77 & 33.22 & 40.77 & 53.54 & 215.00 & 49.33 & 26.94 & 0.55 \\
\hline$a / d(-)$ & 3.50 & 2.50 & 2.99 & 3.40 & 3.50 & 6.00 & 3.37 & 0.66 & 0.20 \\
\hline$b_{w}(\mathrm{~mm})$ & 255.00 & 55.00 & 101.00 & 150.00 & 200.00 & 310.00 & 151.96 & 57.77 & 0.38 \\
\hline$d(\mathrm{~mm})$ & 837.75 & 85.25 & 165.50 & 251.00 & 333.50 & 923.00 & 264.15 & 160.46 & 0.61 \\
\hline
\end{tabular}

* SD denotes standard deviation, C.o.V denotes the coefficient of variation, Max denotes maximum, Min denotes minimum.

\subsection{Preparation of testing and training database}

The construction of an efficient machine learning-based shear strength model for SFRC beams requires the addition of the critical parameters affecting shear strength as input variables. Various studies [1216 , identified the ratio of the shear span to effective depth $a / d$, beam effective depth $d$, beam width $b_{w}$, amount of longitudinal reinforcement $\rho_{l}$, concrete strength $f_{c}$, aggregate size $d_{a}$ and the steel fiber factor $F_{s f}$ as essential variables influencing the shear resistance of SFRC beams. For this reason, the mentioned parameters are considered as input parameters for the development of the ML-based models for this study.

In order to develop and implement the ML algorithms, the experimental evaluation database was split into two subsets: a training and testing database. The training database is adopted to develop both the GP and the RF models, whereas the testing database is utilised to appraise the performance of the proposed predictive models. It was ensured, as much as possible, that the input variables of the training and the testing data patterns are statistically consistent while dividing the database into subsets. $75 \%$ of the evaluation database (244 out of 326 tests) are utilised for training, and the remainder (82 tests) are employed for model testing. Table 2 provides a summary of the input and output parameters for the testing and training database. The geometrical properties are defined by $d, b_{w}$ and the $a / d$. The geometry of a typical beam specimen can be seen in Fig. 3. The properties of the steel reinforcement are described as the percentage of steel reinforcement $\rho_{l}$. The concrete strength $f_{c}$ and the aggregate size $d_{a}$ describes the concrete properties. The attributes of the fibers are defined by the tensile strength of fiber $f_{t}$ and the steel fiber factor $F_{s f}$ (which is a function of the percentage volume $V_{f}$, diameter $d_{f}$ and length of fibers $L_{f}$ ). The shear capacity $V_{u}$ of the SFRC beams is considered as the single output variable.

\subsection{Implementation of the machine learning algorithms}

The algorithms of the Gaussian Process and the Random Forest techniques were implemented in a MATLAB environment. The algorithms were trained using the training evaluation database summarised in Table 2. Each specimen in the training database contains eight input variables and one output variable (shear strength). The objective of implementing the ML algorithms is to develop a model which reasonably predicts the target value (shear capacity) of the test cases in the testing database given only the input variables.

Various types of kernel functions can be used in Gaussian Process regression models. Considering that specific kernel functions are suitable for a given dataset type, four GP kernel functions are considered in this investigation. The investigated kernel functions include the squared exponential kernel (Gaussian/RBF) (Eq. (9)), exponential kernel (Eq. (10)), Matern 5/2 (Eq. (11)) and rational quadratic kernel (Eq. (12)). In order to avoid parameters in higher numeric ranges dominating those in smaller numeric ranges, the data were normalised using the Z-score normalisation technique. In the Z-score method, the values are normalised based on the mean $\mu_{X}$ and standard deviation $\sigma_{X}$ of the data set of the input variable $X$ (e.g. concrete strength). For $X_{i}$ value of input variable $X$, the normalised value is given as $U_{i}=\frac{X_{i}-\mu_{X}}{\sigma_{X}}$. Normalisation helps to avoid numerical problems during the calculation. In this investigation, a 10-fold cross-validation approach is considered

The implementation of the GP and RF algorithms in this study requires the proper selection of the algorithm hyperparameters. The hyperparameters for the GP regression model includes the length parameter $l$, the standard deviation of the noise $\sigma_{n}$ and the covariance $\sigma_{f}$. In the case of the Random Forest model, the design parameters include the depth of the tree; the number of features, seeds, iterations, execution slots and the batch size. The identification of the optimal/best values of the design parameters is majorly a trial and error process. Therefore, the optimum parameters were obtained using the optimum search method.

\subsection{Predictive model performance evaluation metrics}

4.4.1. Statistical parameters - Root-mean-square error (RMSE), mean absolute error (MAE) and coefficient of determination $\left(R^{2}\right)$

The performance of the developed ML-based models is investigated in terms of various statistical parameters such as $R^{2}$, MAE and RMSE. The best model is the one $R^{2}$ value closest to one along with the minimum values of MAE and RMSE (nearest value to zero). The MAE value is a performance metric measured by taking the average of the absolute difference between actual values and the predictions. The function is expressed in Eq. (10).

$M A E=\left(\frac{\sum_{i=1}^{n}\left|R_{\text {pred }}-R_{\text {exp }}\right|}{n}\right)$

The coefficient of determination $R^{2}$ measures how well the regression line replicates the actual outcomes. When the $R^{2}=1$, then a perfect fit is obtained between the predicted and the true/actual values. The mathematical representation for $R^{2}$ is expressed as Eq. (18).

$R^{2}=1-\frac{\sum_{i=1}^{n}\left(R_{\text {pred }}-R_{\text {exp }}\right)^{2}}{\sum_{i=1}^{n}\left(V_{\text {pred }}\right)^{2}}$

The RMSE is obtained as the square root of the average of squared errors obtained as the difference between the predicted and the measured values. The RMSE is determined as Eq. (19).

$R M S E=\sqrt{\frac{1}{n} \sum_{i=1}^{n}\left(R_{\text {pred }}-R_{\text {experiment }}\right)^{2}}$

where $R_{\text {exp }}$ and $R_{\text {pred }}$ are experimental and predicted values. $n$ is the number of data samples

\subsubsection{Predictive model error (model uncertainty)}

The developed models are assessed in terms of the mean (bias) and standard deviation (scatter) of the model error or model uncertainty. 


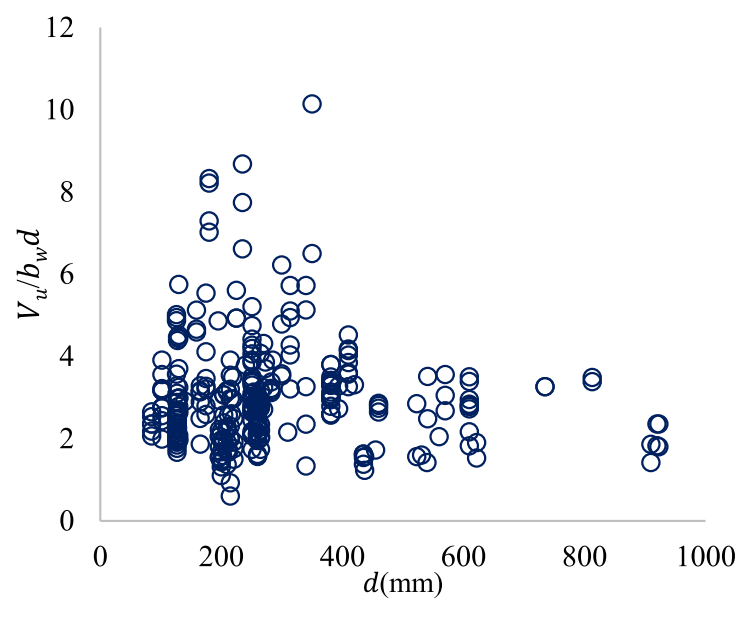

a) Beam effective depth $d$

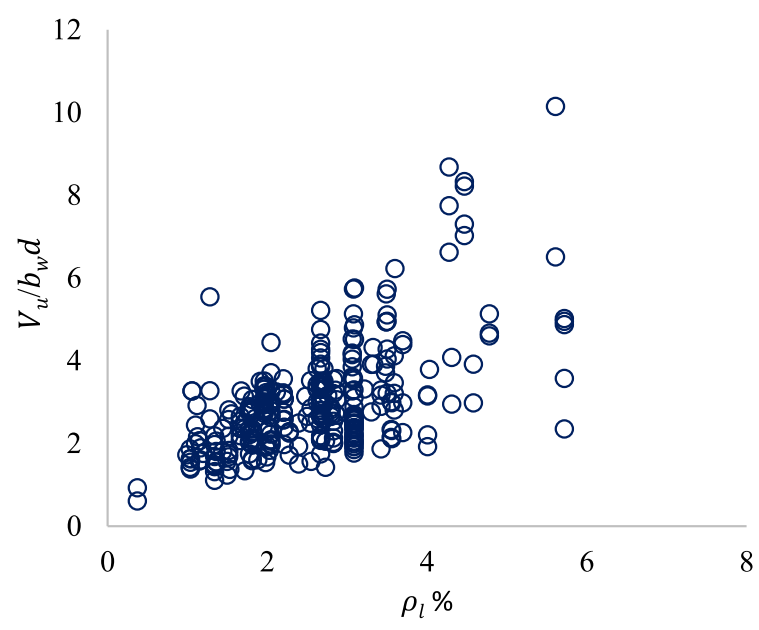

c) Percentage longitudinal reinforcement $\rho_{l} \%$

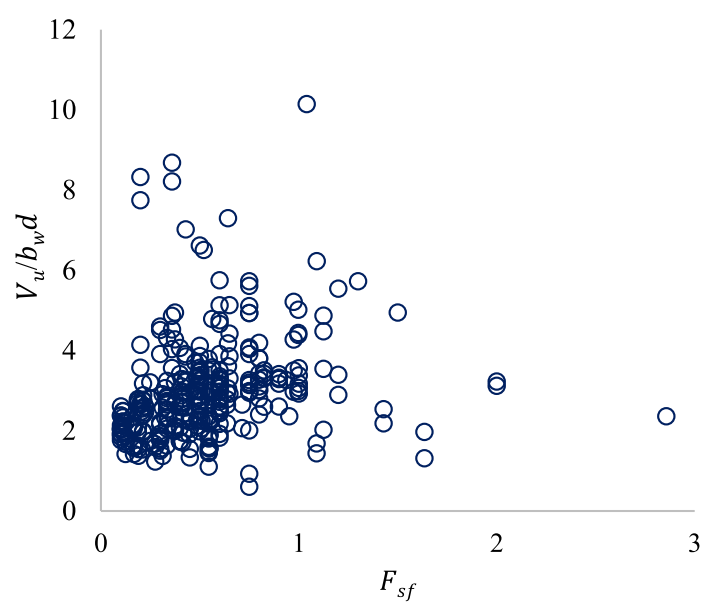

e) Steel fiber factor $F_{s f}$

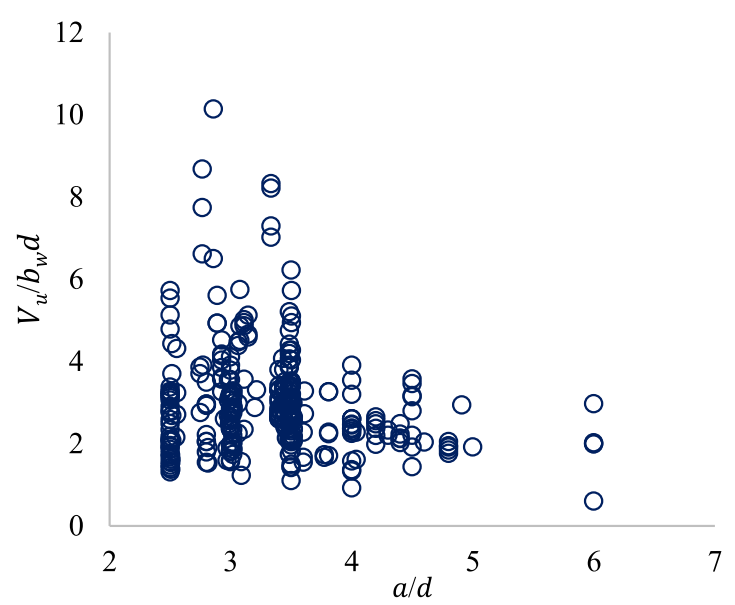

b) Shear -span to effective depth ratio $a / d$

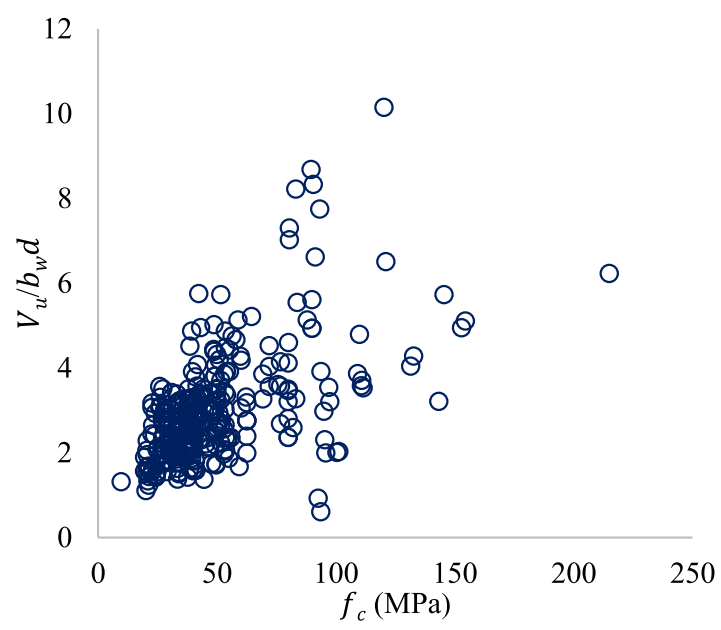

d) Concrete strength $f_{c}$

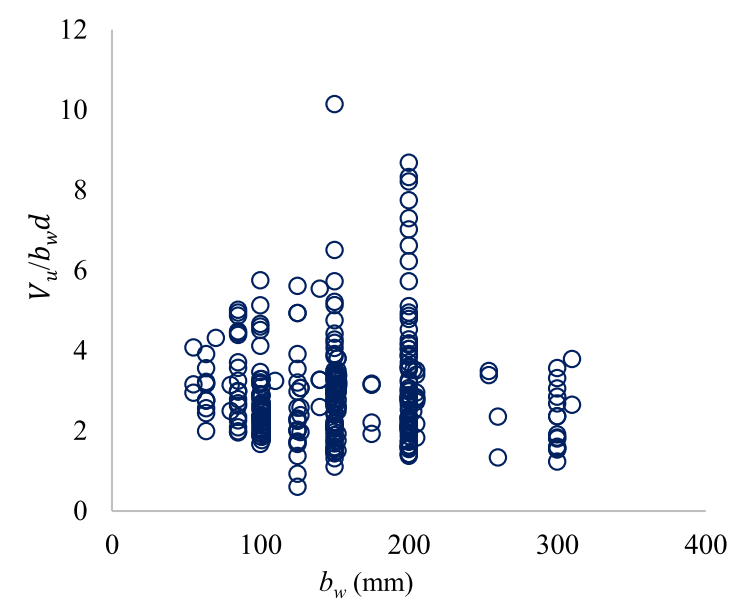

f) beam width

Fig. 2. Shear capacity $V_{u}$ of the SFRC beams as a function of the main shear parameters.

Model uncertainty is described as the deficiency of a model to accurately represent and express a physical phenomenon (in this study, the shear strength) due to lack of understanding of the problem, conservative assumptions and mathematical simplifications $[2,66]$. In structural reliability framework, the model uncertainty is expressed as a random variable with a mean value, standard deviation and probability distribution. It has been shown to have a major impact on shear reliability analysis $[67,68]$. For the predictive models considered in this study, the 
Table 2

Statistical summary of the training and testing dataset.

\begin{tabular}{|c|c|c|c|c|c|c|}
\hline Parameters & Range & Min & Max & Mean & Standard Deviation & Coefficient of Variation \\
\hline \multicolumn{7}{|c|}{ Training dataset (244) } \\
\hline$V_{u}(\mathrm{kN})$ & 686.64 & 12.89 & 699.53 & 133.98 & 128.70 & 0.96 \\
\hline$F_{s f}(-)$ & 2.75 & 0.11 & 2.86 & 0.60 & 0.40 & 0.66 \\
\hline$f_{t}(\mathrm{MPa})$ & 4653.00 & 260.00 & 4913.00 & 1263.69 & 475.73 & 0.38 \\
\hline$d_{a}(\mathrm{~mm})$ & 21.60 & 0.40 & 22.00 & 9.94 & 5.22 & 0.53 \\
\hline$\rho_{l}$ & 0.05 & 0.00 & 0.06 & 0.03 & 0.01 & 0.38 \\
\hline$f_{c}(\mathrm{MPa})$ & 205.23 & 9.77 & 215.00 & 49.35 & 26.89 & 0.54 \\
\hline$a / d(-)$ & 3.50 & 2.50 & 6.00 & 3.37 & 0.66 & 0.20 \\
\hline$b_{w}(\mathrm{~mm})$ & 255.00 & 55.00 & 310.00 & 151.75 & 57.75 & 0.38 \\
\hline$d(\mathrm{~mm})$ & 837.75 & 85.25 & 923.00 & 309.73 & 163.76 & 0.53 \\
\hline \multicolumn{7}{|c|}{ Testing database (82) } \\
\hline$V_{u}(\mathrm{kN})$ & 701.92 & 18.61 & 720.53 & 162.40 & 126.65 & 0.78 \\
\hline$F_{s f}(-)$ & 2.75 & 0.11 & 2.86 & 0.60 & 0.40 & 0.66 \\
\hline$f_{t}(\mathrm{MPa})$ & 2340.00 & 260.00 & 2600.00 & 1293.89 & 472.35 & 0.37 \\
\hline$d_{a}(\mathrm{~mm})$ & 21.60 & 0.40 & 22.00 & 11.84 & 4.10 & 0.35 \\
\hline$\rho_{l}$ & 0.05 & 0.01 & 0.06 & 0.03 & 0.01 & 0.36 \\
\hline$f_{c}(\mathrm{MPa})$ & 125.95 & 19.60 & 145.55 & 47.92 & 24.21 & 0.51 \\
\hline$a / d(-)$ & 3.50 & 2.50 & 6.00 & 3.29 & 0.58 & 0.18 \\
\hline$b_{w}(\mathrm{~mm})$ & 245.00 & 55.00 & 300.00 & 160.61 & 48.60 & 0.30 \\
\hline$d(\mathrm{~mm})$ & 824.75 & 85.25 & 910.00 & 309.73 & 163.76 & 0.53 \\
\hline
\end{tabular}

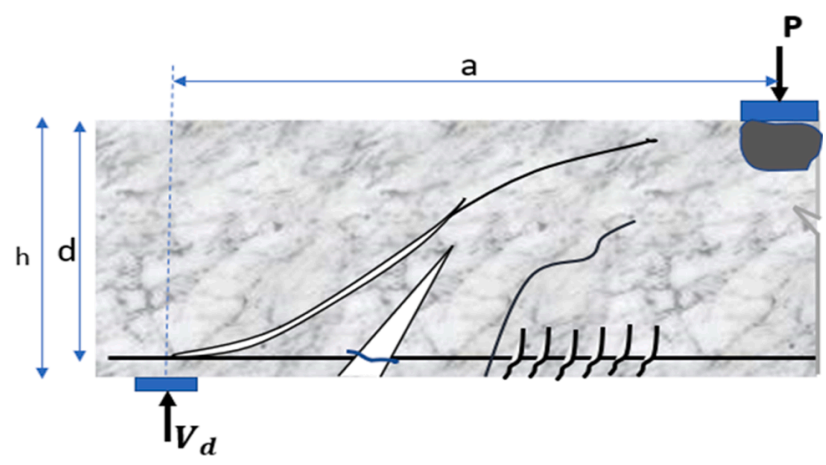

Fig. 3. Geometry of a typical beam specimen.

model uncertainty (or model error) associated with a single specimen $x$ is defined as the ratio of the experimental to predicted shear strength, expressed in Eq. (20) $[66,67]$.

$M_{x}=\frac{R_{\text {exp }}}{R_{\text {pred }, x}(X)}$

$M\left(\mu_{M}, \sigma_{M}\right)$

where, $M_{x}$ is the model uncertainty for a single beam test $x$ in shear. $X$ represents the input variables for the predictive model $\left(a / d, d, b_{w}, \rho_{l}\right.$, $f_{c}, f_{t}, d_{a}$ and $\left.F_{s f}\right) . \mu_{M}$ and $\sigma_{M}$ represents the mean and standard deviation of the model uncertainty variable, respectively.

The best model is the one with model uncertainty mean value $\mu_{M}$ closest to one along with the minimum standard deviation $\sigma_{M}$ (nearest value to zero). $\mu_{M}>1$ implies that the model predicts a lower value of ultimate shear capacity than the failure load of the beam specimen and thus underpredicts. Conversely, $\mu_{M}<1$ implies that it overpredicts the actual shear resistance.

\section{Results and discussion}

\subsection{ML-based predictive models: Performance evaluation}

In this study, four kernel functions (Squared exponential kernel, Exponential kernel, Matern 5/2 and Rational quadratic kernel) were trialled to develop the most reasonable GPR model. The optimum parameters for the kernel functions (the length parameter $l$, standard deviation of the noise $\sigma_{n}$ and the covariance $\sigma_{f}$ ) were obtained using the optimum search method. The performance of the developed GPR model using the training and testing database, and for various kernel functions is presented in Table 3. As shown in the table, all the kernel functions exhibited a reasonable degree of accuracy in predicting the shear strength of SFRC beams. It is evident that the rational quadratic kernel produced the best performance with $R^{2}=0.94$ and the lowest RMSE and MAE scores (32.68 and 19.23 respectively) using the training data. Similarly, $R^{2}$, RMSE and MAE values of $0.94,30.8$ and 22.4 is obtained for the testing database, respectively. Hence further discussions in this study will be based on the GPR rational quadratic model. The statistical results of the developed Random Forest predictive model are presented in Table 4. Compared to the GPR models, the RF model displayed better performance in terms of the $R^{2}$ of the training and testing database. The RMSE and MAE values from both the GPR and RF models are comparable.

To further demonstrate the efficiency of the proposed models (GPR and RF), the plots of their predicted shear resistance versus experimentally observed shear strength for the training and testing database are presented in Figs. 4 and 5 respectively. As shown on the figures, the predictions of the proposed models match well with the experimentally observed strength. A high prediction capability was obtained by the testing database even though it was not used for the training of the models. This demonstrates the generalisation capability of the proposed models. As the value of the shear strength increases, the scatter becomes more significant due to the reduction in the number of observations. Fig. 6 presents a head-to-head plot of the predicted and experimental shear resistance against the record number of observations (testing

Table 3

GPR model performance for different kernel functions.

\begin{tabular}{|c|c|c|c|c|c|c|}
\hline \multirow[t]{2}{*}{ Model } & \multicolumn{3}{|c|}{ Training dataset } & \multicolumn{3}{|c|}{ Testing dataset } \\
\hline & RMSE & $R^{2}$ & $M A E$ & RMSE & $R^{2}$ & $M A E$ \\
\hline Rational Quadratic & 32.68 & 0.94 & 19.23 & 30.8 & 0.94 & 22.4 \\
\hline Matern5/2 & 32.83 & 0.94 & 19.36 & 30.6 & 0.94 & 22.4 \\
\hline Exponential & 33.93 & 0.93 & 19.8 & 33.48 & 0.93 & 23.5 \\
\hline Gaussian RBF & 34.51 & 0.93 & 20.9 & 31.28 & 0.94 & 23 \\
\hline
\end{tabular}


Table 4

RF model performance.

\begin{tabular}{rllllll}
\hline \multirow{2}{*}{ Model } & \multicolumn{2}{l}{ Training dataset } & \multicolumn{4}{l}{ Testing dataset } \\
& $R M S E(k N)$ & $R^{2}$ & $M A E(k N)$ & $R M S E(k N)$ & $R^{2}$ & $M A E(k N)$ \\
Random & 39.4 & 0.95 & 21.9 & 37.32 & 0.96 & 23.03 \\
Forest & & & & & & \\
\hline
\end{tabular}

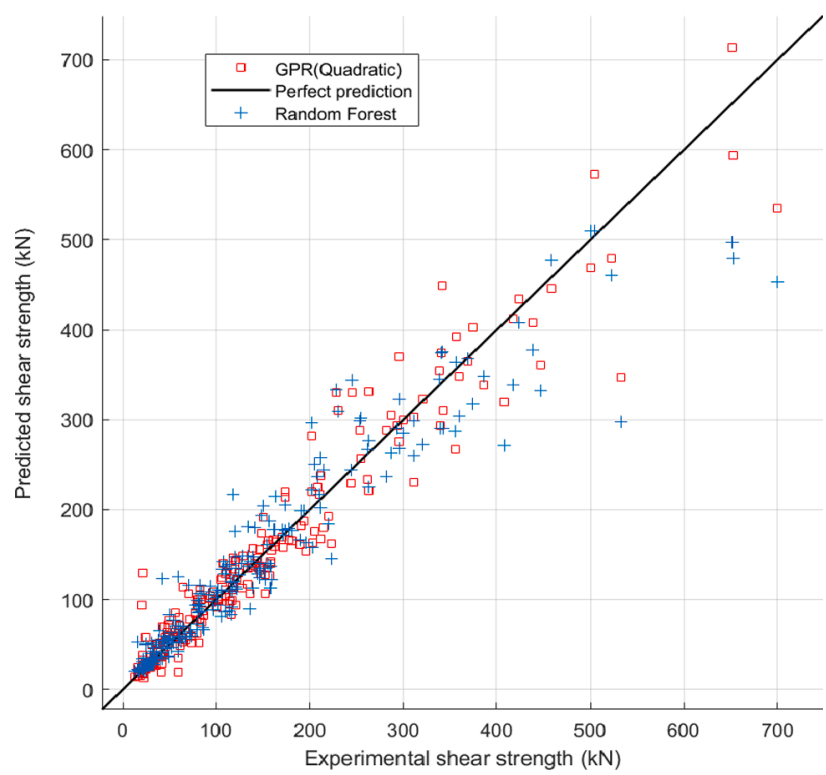

Fig. 4. Plot of predicted shear capacity $(\mathrm{kN})$ against experimental shear capacity $(\mathrm{kN})$ using the training database.

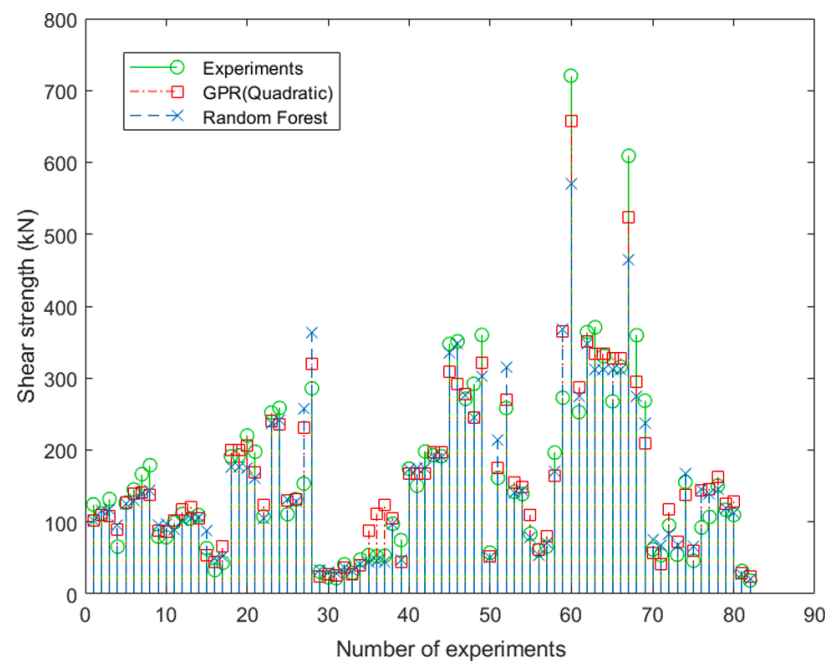

Fig. 5. Comparison of predictions from the GPR (rational quadratic) and RF to the experimental values using STEM plot (testing database).

database). From the chart, it can be noticed that the trends by the developed models have a good resemblance and a minimal mismatch with the trendline of experimental results for the testing datasets (see Fig. 6).

\subsection{Comparative assessment of the developed ML-based models and existing SFRC shear strength models}

A comparative assessment is carried out to evaluate the predictive capability of the developed ML-based models (GPR and RF) against

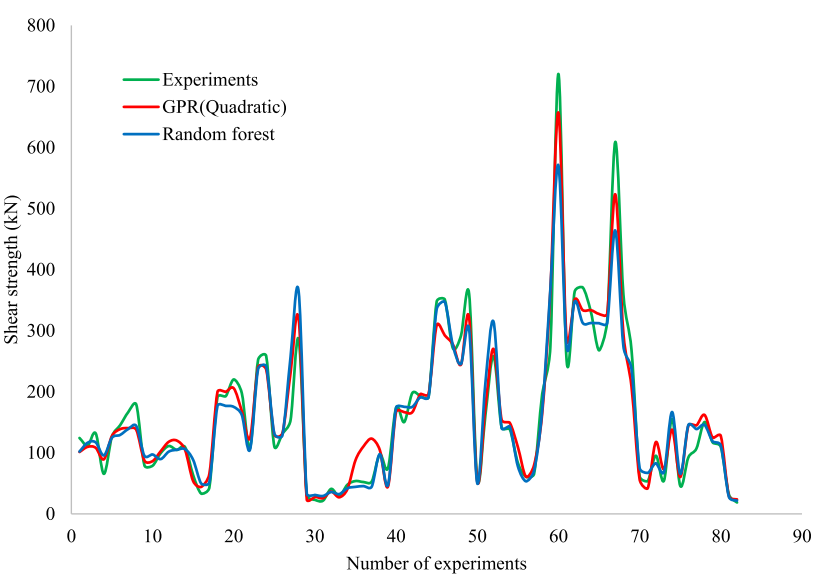

Fig. 6. Comparison of predictions from the GPR (Quadratic) and RF to the experimental values using the testing dataset.

existing shear resistance predictive models for SFRC beams. The existing shear strength models considered in this study include the code equations from Fib Model Code 2010 (MC-10) (Eq. (4)) and the German guideline (DAfStB) (Eq. (1)) and the recently developed modified MASM equation (M-MASM) (Eq. (5)). The included safety factors/bias used in the code equations were removed (taken as 1) when the models were evaluated against each other and the experimental database. A plot of the correlation between the experimentally observed shear strength $V_{\exp }$ and the predicted shear strength $V_{\text {pred }}$ for the five considered models (GPR, RF, MC-10, DAfStB and M-MASM), using the testing evaluation database is presented in Fig. 7. A tolerance of $\pm 20 \%$ is shown on the graph. Generally, the predicted capacity by the various methods deviate from the 'perfect line' (defined as the point where $V_{\text {exp }}=V_{\text {pred }}$ ). As shown on the plot, the predictions from the GPR model are less dispersed compared to other methods and mostly lie within $\pm 20 \%$ line of equality/perfect line.

A statistical comparison of the proposed ML-based shear strength models in terms of the model uncertainty statistics (mean and coefficient of variation), $R^{2}$, RMSE and MAE, using the testing database, are summarised in Table 5. A comparison of the ML-based models with the existing design models in terms of RMSE and MAE results is illustrated in Fig. 8. From Table 5 and Fig. 8, it is observed that the ML-based models have the highest $R^{2}$ values and the lowest RMSE and MAE values of all the models investigated. The respective uncertainty distributions are shown in a box plot presented in Fig. 9. The model uncertainty variable

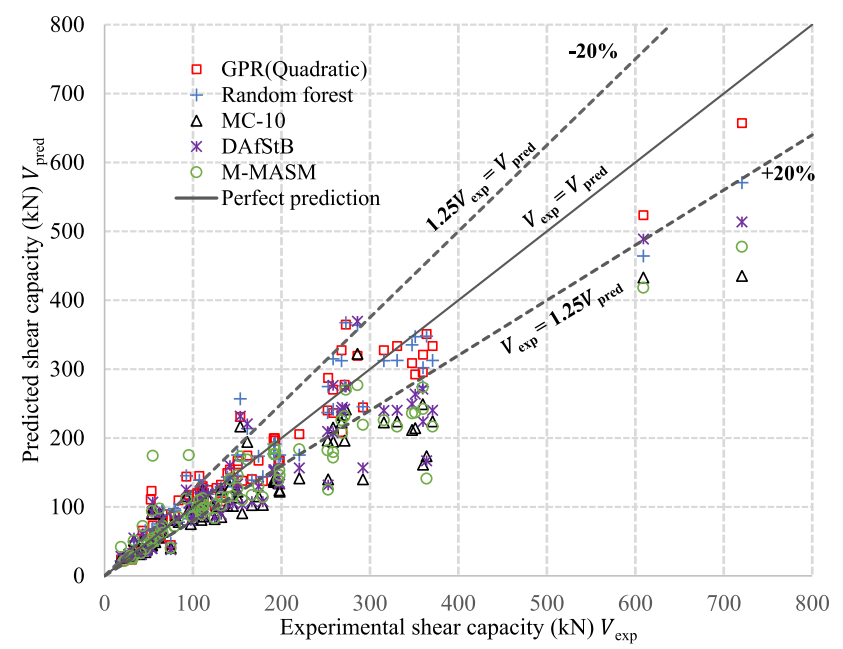

Fig. 7. Predicted value versus the experimental shear capacities for the developed models and existing method. 
Table 5

Statistical properties of the developed and existing model (using the testing data set).

\begin{tabular}{llllll}
\hline Parameters & GPR & RF & MC-10 & DAfStB & M-MASM \\
\hline$R^{2}$ & 0.94 & 0.95 & 0.86 & 0.86 & 0.87 \\
RMSE & 32.68 & 39.4 & 70.8 & 58.6 & 64.4 \\
MAE & 19.23 & 21.9 & 46.82 & 39.9 & 40.2 \\
Mean $\mu_{M}$ & 0.98 & 1.00 & 1.24 & 1.17 & 1.17 \\
Standard deviation $\sigma_{M}$ & 0.20 & 0.18 & 0.35 & 0.32 & 0.33 \\
Coefficient of variation $V_{M}$ & 0.20 & 0.18 & 0.28 & 0.27 & 0.28 \\
Skewness $\left(\eta_{M}\right)$ & 0.03 & 0.01 & 0.38 & 0.45 & 0.75 \\
Range & 1.25 & 0.98 & 1.68 & 1.67 & 2.27 \\
Minimum $\left(M_{\min }\right)$ & 0.43 & 0.60 & 0.55 & 0.51 & 0.31 \\
Maximum $\left(M_{\max }\right)$ & 1.68 & 1.58 & 2.2 & 2.17 & 2.58 \\
\hline
\end{tabular}

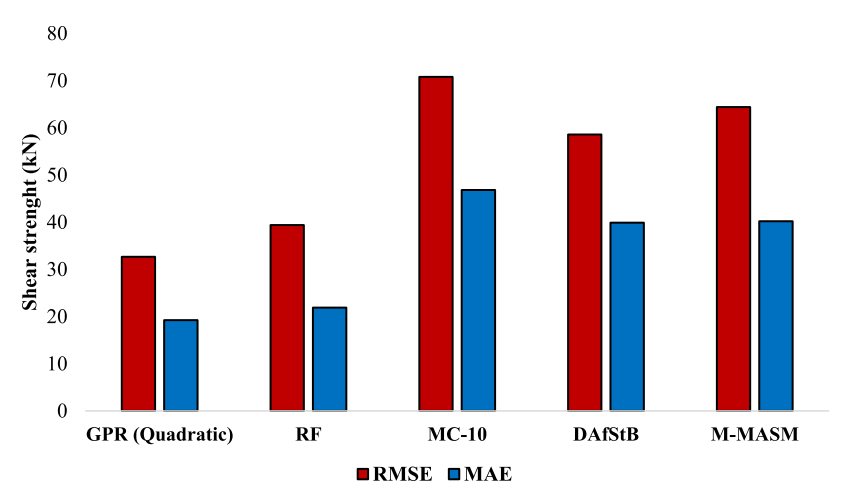

Fig. 8. Comparison of the developed models with existing models in terms of RMSE and MAPE.

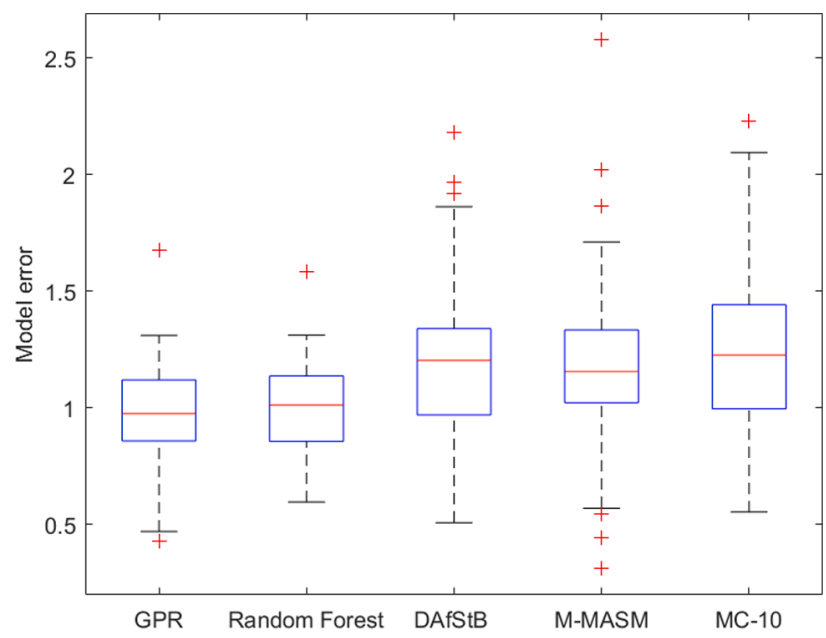

Fig. 9. Box plot of model error distributions.

related to the RF model has a mean value of $\mu_{M}=\frac{V_{\text {exp }}}{V_{\text {pred }}}=1.00$ (closest to the optimal value of 1 ). Concerning dispersion, the RF model produces the lowest standard deviation of all the models investigated with $\sigma_{M}=$ 0.18 . The MC-10 shear model has the highest mean value of $\mu_{M}=1.24$ (largest conservative bias) and the most substantial dispersion with $\sigma_{M}=0.35$. Table 6 presents the model error statistics obtained for MC10 , DAfStB and M-MASM from previous studies using the database compiled by [15] containing both slender and non-slender SFRC beams. The same database was used for the assessment conducted in this study with the exclusion of non-slender beams. The model uncertainty characteristics obtained for existing models investigated in this study are comparable to those recorded by other researchers listed in Table 6 .
Table 6

Model error statistics from literature.

\begin{tabular}{lllll}
\hline Model & Source & Mean $\left(\mu_{M F}\right)$ & $\begin{array}{l}\text { SD } \\
\left(\sigma_{M F}\right)\end{array}$ & $\begin{array}{l}\text { Sample } \\
\text { size \# }\end{array}$ \\
\hline $\begin{array}{l}\text { Fib Model Code } \\
\text { (MC-10) }\end{array}$ & $\begin{array}{l}{[18] \text { (Slender and non- }} \\
\text { slender beams) } \\
\text { DAfStB }\end{array}$ & 1.24 & 0.36 & 488 \\
M-MASM & $\begin{array}{l}\text { slender beams) } \\
\text { slender (Slender and non- } \\
\text { slender beams) }\end{array}$ & 1.12 & 0.31 & 488 \\
& 1.15 & 0.25 & 488 \\
\hline
\end{tabular}

Considering the statistical parameters $R^{2}$, model error mean value $\mu_{M}$ and standard deviation $\sigma_{M}$, the overall precision of the RF model exceeds that of the GPR as well as the existing models investigated. Although the accuracy of the GPR model is higher than that of the existing models.

In order to establish the most suitable predictive model, a rationalised ranking method for predictive models, introduced by Olalusi \& Spyridis [69] is adopted in this study. According to this method, the models are ranked, based on the following criteria:

i. Best ranking for the highest $R^{2}$

ii. Best ranking for least RMSE

iii. Best ranking for least MAE

iv. Best ranking for mean model error $\mu_{M}$ closest to the optimal value of 1

v. Best ranking for least standard deviation $\sigma_{M}$

vi. Best ranking for the minimum ratio of $\left(M_{\max } / M_{\min }\right)$

For each criterion, the five models investigated are awarded ranking scores from 1 to 5 , starting with the model with the best ranking. The overall ranking is the average of ranking scores from criteria i-vi. Based on this system, the developed RF model ranks first, followed by the GPR model, the DAfStB model, the M-MASM and the MC-10 model (Table 7).

\subsection{Influence of input variables $X_{i}$ on the model performance (sensitivity analysis)}

A sensitivity analysis is undertaken to measure the significance of the input parameters in predicting the shear strength of SFRC beams. The sensitivity of the input parameters is assessed by omitting each input variable from the database once at a time and using the resultant dataset to train and test the proposed models. The significance of each variable on the shear resistance of SFRC beams was evaluated using by $R^{2}$, RMSE and MAE values of the training and testing database and the results presented in Table 8. Since the rational quadratic kernel yielded the best performance for the GP regression model, the sensitivity analysis of the model was conducted using the rational quadratic kernel. The table shows that all the input variables considered to affect the shear strength of SFRC beams. However, compared to other input variables, the beam depth, beam width and concrete strength are the most sensitive variables affecting the shear strength of SFRC beams. It can be noted that omitting the tensile strength of fiber $f_{t}$ results in a minimal change in the performance of the developed models.

Table 7

Predictive model's ranking system.

\begin{tabular}{llllll}
\hline Parameters & GPR & RF & MC-10 & DAfStB & M-MASM \\
\hline Ranking for $R^{2}$ & 2 & 1 & 3 & 3 & 4 \\
Ranking for RMSE & 1 & 2 & 5 & 3 & 4 \\
Ranking for MAE & 1 & 2 & 5 & 3 & 4 \\
Ranking for $\mu_{M}$ & 2 & 1 & 4 & 3 & 3 \\
Ranking for $\sigma_{M}$ & 2 & 1 & 5 & 3 & 4 \\
Ranking for $\left(M_{\max } / M_{\min }\right)$ & 2 & 1 & 3 & 4 & 5 \\
Overall ranking & 1.7 & 1.3 & 4.2 & 3.2 & 4.0 \\
\hline
\end{tabular}


Table 8

Impact of input variables on the performance of the proposed ML models.

\begin{tabular}{|c|c|c|c|c|c|c|c|}
\hline \multirow[t]{2}{*}{ Model } & \multirow[t]{2}{*}{ Input variables } & \multicolumn{3}{|c|}{ Training dataset } & \multicolumn{3}{|c|}{ Testing dataset } \\
\hline & & $R M S E(k N)$ & $R^{2}$ & $M A E(k N)$ & $R M S E(k N)$ & $R^{2}$ & $M A E(k N)$ \\
\hline \multirow[t]{9}{*}{ GPR } & $F_{s f}, f_{t}, f_{c} d_{a}, \rho_{l}, a / d, b_{w}, d$ & 32.68 & 0.94 & 19.23 & 30.8 & 0.94 & 22.4 \\
\hline & $F_{s f}, f_{t}, f_{c} d_{a}, \rho_{l}, a / d, d$ & 45.55 & 0.88 & 28.17 & 36.9 & 0.92 & 26.0 \\
\hline & $F_{s f}, f_{t}, f_{c} d_{a}, \rho_{l}, a / d, b_{w}$ & 50.01 & 0.85 & 28.91 & 51.3 & 0.84 & 35.52 \\
\hline & $F_{s f}, f_{t}, f_{c} d_{a}, \rho_{l}, b_{w}, d$ & 37.6 & 0.92 & 23.4 & 33.09 & 0.94 & 23.4 \\
\hline & $F_{s f}, f_{t}, f_{c} d_{a}, a / d, b_{w}, d$ & 40.34 & 0.90 & 23.1 & 29.8 & 0.95 & 21.2 \\
\hline & $F_{s f}, f_{t}, f_{c} \rho_{l}, a / d, b_{w}, d$ & 36.3 & 0.92 & 20.8 & 31.2 & 0.94 & 22.3 \\
\hline & $F_{s f}, f_{t}, d_{a}, \rho_{l}, a / d, b_{w}, d$ & 39.72 & 0.91 & 25.2 & 40.7 & 0.89 & 27.6 \\
\hline & $F_{s f}, f_{c} d_{a}, \rho_{l}, a / d, b_{w}, d$ & 34.9 & 0.93 & 19.9 & 31.8 & 0.94 & 22.1 \\
\hline & $f_{t}, f_{c} d_{a}, \rho_{l}, a / d, b_{w}, d$ & 37.3 & 0.92 & 23.1 & 32.2 & 0.92 & 23.3 \\
\hline \multirow[t]{9}{*}{$\mathrm{RF}$} & $F_{s f}, f_{t}, f_{c} d_{a}, \rho_{l}, a / d, b_{w}, d$ & 39.4 & 0.95 & 21.9 & 37.32 & 0.96 & 23.03 \\
\hline & $F_{s f}, f_{t}, f_{c} d_{a}, \rho_{l}, a / d, d$ & 48.6 & 0.93 & 27.2 & 40.5 & 0.95 & 23.8 \\
\hline & $F_{s f}, f_{t}, f_{c} d_{a}, \rho_{l}, a / d, b_{w}$ & 53.2 & 0.91 & 29.0 & 49.5 & 0.93 & 31.7 \\
\hline & $F_{s f}, f_{t}, f_{c} d_{a}, \rho_{l}, b_{w}, d$ & 40.9 & 0.95 & 22.8 & 35.4 & 0.96 & 22.8 \\
\hline & $F_{s f}, f_{t}, f_{c} d_{a}, a / d, b_{w}, d$ & 41.9 & 0.95 & 23.3 & 36.6 & 0.96 & 23.3 \\
\hline & $F_{s f}, f_{t}, f_{c} \rho_{l}, a / d, b_{w}, d$ & 39.8 & 0.95 & 21.7 & 37.3 & 0.95 & 22.6 \\
\hline & $F_{s f}, f_{t}, d_{a}, \rho_{l}, a / d, b_{w}, d$ & 47.5 & 0.93 & 27.2 & 40.2 & 0.95 & 27.0 \\
\hline & $F_{s f}, f_{c} d_{a}, \rho_{l}, a / d, b_{w}, d$ & 40.2 & 0.95 & 22.3 & 37.3 & 0.96 & 23.0 \\
\hline & $f_{t}, f_{c} d_{a}, \rho_{l}, a / d, b_{w}, d$ & 42.6 & 0.95 & 23.5 & 38.7 & 0.95 & 25.47 \\
\hline
\end{tabular}
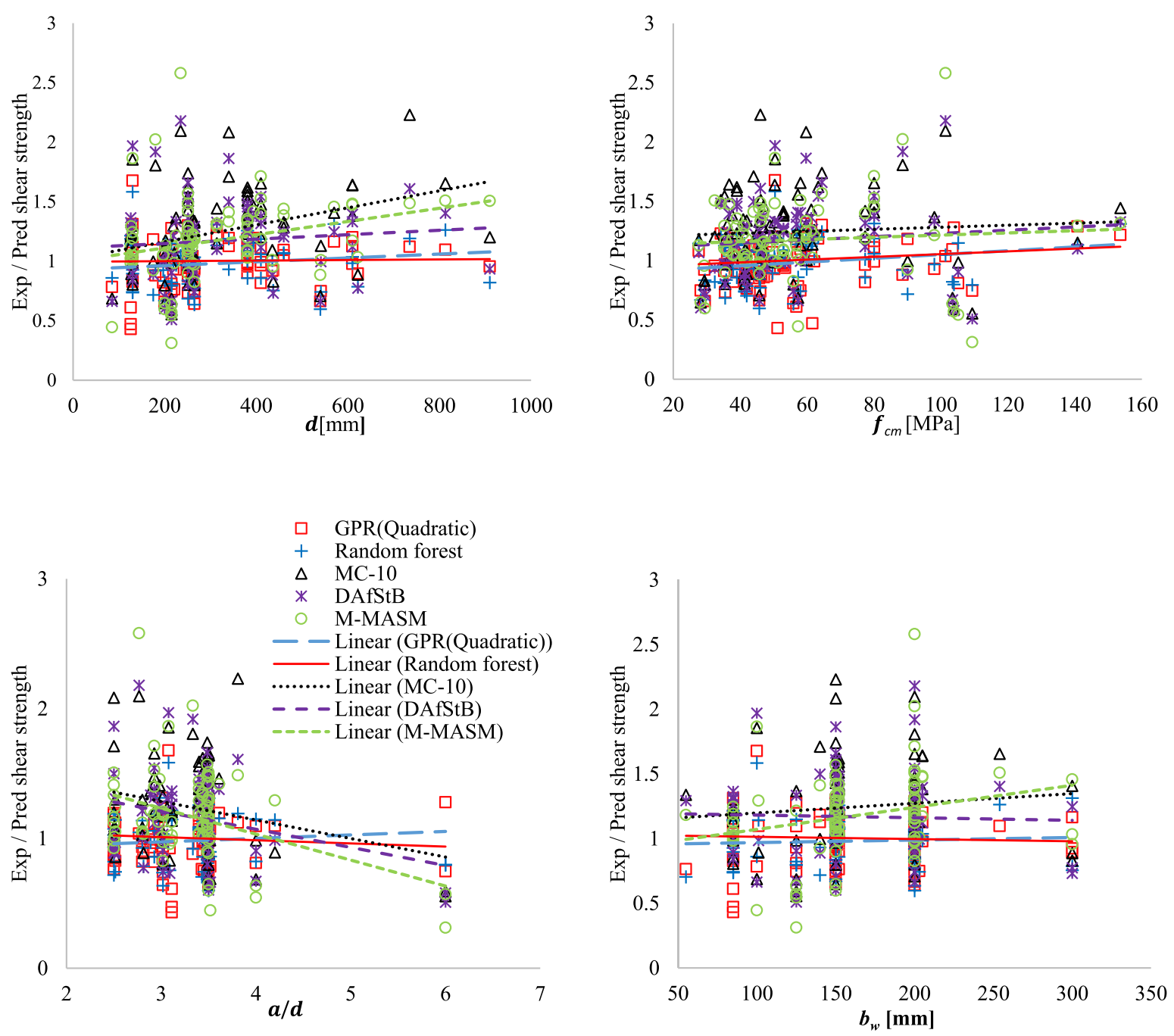

Fig. 10. Relationship between the ratio of experimental to predicted shear strength versus shear parameters. 


\subsection{Trends in the model error with input variables $X_{i}$; parametric performance assessment}

The trend in the performance of the various models with input variables (shear parameters) is assessed by correlating the prediction model error (model uncertainty) $M_{x}$ with important shear input variables $X_{i}$ (shear span to depth ratio $a / d$, beam effective depth $d$, beam width $b_{w}$ and concrete strength $f_{c}$ ). The variation and trends of the model error $M_{x}$ with the basic input variables $X_{i}$ are shown in Fig. 10, using the testing database. The trend analysis allows us to discern any trend/bias in the performance of the various models in relation to their input variables.

As observed from the figures, the accuracy of the results of the developed ML-based models does not show any significant and apparent trend or bias with the shear span to effective depth ratio, beam effective depth, beam width and the concrete strength, for the range investigated in this study. This means that the proposed models can be used with acceptable confidence for all the range of shear parameters considered in this study. On the other hand, the existing methods (M-MASM, MC10 and DAfStB) have some trend with the $d$ and $a / d$. The strong decreasing trend/safety bias observed as the shear span to effective depth ratio $a / d$ increases is a cause for concern. Reliability evaluation of the shear design formulations is advised at parametric ranges of $a / d$.

\subsection{Derivation of a partial factor for the model uncertainty related to shear resistance predictive models using deterministic reliability verification}

Resistance partial factor $\gamma_{R d}$ can be used to account for the uncertainties related to resistance models $[70,71]$. Resistance partial factors accounting for the model uncertainty related to M-MASM, MC-10 and DAfStB shear resistance models, using model uncertainty $M$ statistics presented in Table 5, are proposed in this section. The partial factor $\gamma_{R d}$ is estimated following the procedures established in EN 1990. The model uncertainty random variable $M\left(\mu_{M}, \sigma_{M}\right)$ related to the existing models was taken as a lognormal distribution because the skewness coefficients $\eta_{M}$ of the models (Table 5) are closer to what is required for a lognormal distribution. A lognormal distribution is also recommended for model uncertainty by the JCSS [72]. For a lognormal distribution, the partial factor $\gamma_{R d}$ can be derived using the expression in Eq. (17).

$\gamma_{R d}=1 /\left[\mu_{M} \cdot \exp \cdot\left(-\alpha_{R} \cdot \beta \cdot V_{M}\right)\right]$

where $\alpha_{R}$ denotes the FORM sensitivity factor $=0.8$ [70]. (the model uncertainty is assumed as dominant resistance variable). $\beta$ is the target reliability, according to EN 1990. $\mu_{M}$ and $V_{M}$ are the mean and coefficient of variation of the model uncertainty random variable,

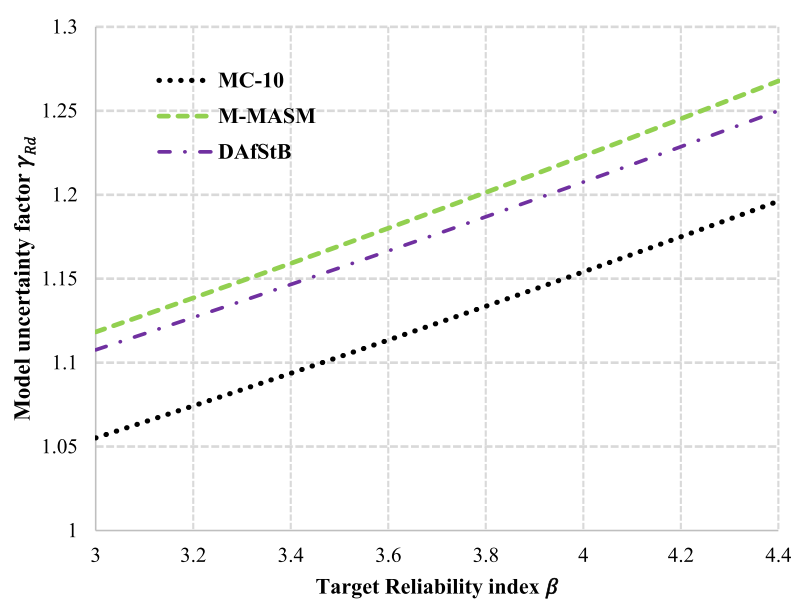

Fig. 11. Variation of the partial factor $\gamma_{R d}$ with. $\beta$ respectively. Fig. 11 presents the variation of the obtained model uncertainty partial factor $\gamma_{R d}$ with target reliability $\beta$ for EN 1992-4. The range of target reliability presented in the figure covers the target reliabilities for 50 years of design working life period for the ultimate limit states for structures. As shown in Fig. 11, the model uncertainty factor $\gamma_{R d}$ increases as the target reliability $\beta$ increases. For the target reliability level of Reliability Class 2 (RC2) structure ( $\beta=3.8$ ), the model uncertainty partial factor $\gamma_{R d}$ obtained for MC-10, M-MASM and DAfStB are $1.13,1.20$ and 1.19 respectively.

According to EN 1990, the design values of the derived model uncertainty is incorporated into the design expressions through the resistance model uncertainty partial factor $\gamma_{R d}$ applied on the total model as expressed in Eq. (18)

$V_{d}=V\left[\eta \frac{X_{k}}{\gamma_{m}} ; a_{d} \cdots\right] / \gamma_{R d}$

where $V_{d}$ is the design resistance. $X_{k}$ is the characteristic values of the variable. $\eta$ is a conversion factor appropriate to the material property. $\gamma_{m}$ is the material property factor. $a_{d}$ is the design geometric parameter. $\gamma_{R d}$ is the resistance model uncertainty factor.

\section{Conclusions}

This contribution proposes efficient machine learning-based methods to predict the ultimate shear capacity of SFRC slender beams without stirrups. The shear behaviour of SFRC beam is a complex phenomenon, involving the interaction of many parameters and mechanism. So far, developing a precise and acceptable design formulation for shear capacity of SFRC beams is still an evolving process. Two machine learning algorithms -Gaussian Process regression and the Random Forest techniques- are applied for the first time to predict the shear capacity of SFRC slender beams. A total of 326 experimental tests were considered for the model development, utilising $75 \%$ for model training and the remainder for testing. Seven input variables $\left(a / d, d, b_{w}, \rho_{l}, f_{c}, d_{a}\right.$ and $\left.F_{s f}\right)$ were considered for constructing the models. The single output variable is the experimentally observed shear strength. The performance of the proposed shear strength models was investigated in terms of statistical parameters such as the $R^{2}$, MAE, RMSE and model uncertainty or model error variable.

From the present contribution, the following general conclusions may be drawn:

- When assessing the performance of the proposed models against experimental results, the predictions from the models match well with the experimental shear capacity. A high prediction capability was obtained by the testing database even though it was not utilised for the training of the models. This demonstrates the generalisation capability of the developed models.

- Sensitivity analysis was carried out to measure the significance of the input variables on the model performance. The sensitivity analysis depicted the beam depth, beam width and concrete strength as the most sensitive variables affecting the performance of the proposed ML-based shear strength models.

- The performance of the proposed ML-based models was investigated as regards their accuracy against the state-of-practice predictive model embedded in international design standards and literature (fib Model Code 2010, German guideline, M-MASM [26]). The best model is the one with the $R^{2}$ value closest to one along with the minimum values of MAE and RMSE (nearest value to zero). Based on these parameters, the overall precision of the ML-based strength models outperforms that of the existing models.

- The model uncertainty or model error random variables associated with the proposed models and the existing models were characterised in this study. The model uncertainty variable related to the RF model has a mean value of $\mu_{M}=1.00$ (closest to the optimal value of 
1) and the lowest dispersion wit. The MC-10 model has the highest mean value of $\mu_{M}=1.24$ (highest conservative bias) and the most substantial dispersion with $\sigma_{M}=0.35$.

- Partial resistance factors accounting for the uncertainties and bias in the existing SFRC shear strength models were proposed. It is important to note that improved estimates of the partial factors can be obtained from a fully probabilistic approach. The derived model uncertainty statistics and related partial safety factors are associated with the specific database considered in this study, which may change noticeably should the database change. The use of partial factors ensures a consistent level of reliability over a range of structures [73].

- Trend analysis was conducted to discern any trend/bias in the performance of the various models concerning their input variables. The accuracy of the results of the proposed ML-based models showed no apparent trend or bias with the shear input variables for the range investigated in this study. The existing methods M-MASM, MC-10 and DAfStB have strong decreasing trend/safety bias as the shear span to effective depth ratio $a / d$ increases. This is a cause for concern. Reliability assessment of the shear design formulations is advised at parametric ranges of shear span to effective depth ratio $a / d$.

- A general probabilistic model for shear reliability analysis should typically be based on a shear predictive model characterised by low bias and uncertainty as well as statistical independence with its input variables [66]. The ML-based shear strength models proposed in this study can be adopted as a general probabilistic model in a simplified reliability framework. This is justified by the fact that they have low bias and uncertainty compared to the other models.

The findings from this investigation are valuable for the development of reliability-based shear design provisions for SFRC beams.

\section{Declaration of Competing Interest}

None.

\section{References}

[1] Pillai S, Kirk D. Reinforced Concrete Design in Canada. McGraw-Hill Ryerson. Limited; 1983.

[2] Olalusi OB. Reliability assessment of shear design provisions for reinforced concrete beams with stirrups (Doctoral dissertation. Stellenbosch: Stellenbosch University); 2018.

[3] Romualdi JP, Batson GB. Mechanics of crack arrest in concrete beam with closely spaced reinforcement. J Am Inst 1963;60:775-89.

[4] Balaguru PN, Shah SP. Fiber-Reinforced Cement Composites, McGraw-Hill, New York; 1992. p. 350.

[5] Babafemi AJ, Kolawole JT, Olalusi OB. Mechanical and Durability Properties of Coir Fiber Reinforced Concrete. J Eng. Sci Technol 2019;14(3):1482-96.

[6] Yazıc1 S, İnan G, Tabak V. Effect of aspect ratio and volume fraction of steel fiber on the mechanical properties of SFRC. Constr Build Mater 2007;21(6):1250-3.

[7] Özcan DM, Bayraktar A, Şahin A, Haktanir T, Türker T. Experimental and finite element analysis on the steel fiber-reinforced concrete (SFRC) beams ultimate behavior. Constr Build Mater 2009;23(2):1064-77.

[8] Gandomi AH, Alavi AH, Yun GJ. Nonlinear modeling of shear strength of SFRC beams using linear genetic programming. Struct Eng Mech 2011:38(1):1-25.

[9] Kang THK, Kim W, Kwak YK, Hong SG. Shear Testing of Steel Fiber-Reinforced Lightweight Concrete Beams without Web Reinforcement. ACI Struct J 2011;108 (5).

[10] Dinh HH, Parra-Montesinos GJ, Wight JK. Shear behavior of steel fiber-reinforced concrete beams without stirrup reinforcement. ACI Struct 2010;597-606. https:// doi.org/10.14359/51663913.

[11] Ahmadi M, Kheyroddin A, Dalvand A, Kioumarsi M. New empirical approach for determining nominal shear capacity of steel fiber reinforced concrete beams. Constr Build Mater 2020;234:117293.

[12] Spinella N, Colajanni P, Recupero A. Simple plastic model for shear critical SFRC beams. J Struct Eng 2010;136(4):390-400.

[13] Arslan G. Shear strength of steel fiber reinforced concrete (SFRC) slender beams. KSCE J Civ Eng 2014;18(2):587-94.

[14] Minelli F. Plain and fiber reinforced concrete beams under shear loading: structural behavior and design aspects. Italy: University of Brescia; 2005.

[15] Lantsoght EO. Database of shear experiments on steel fiber reinforced concrete beams without stirrups. Materials 2019;12(6):917.
[16] Lantsoght EOL. How do steel fibers improve the shear capacity of reinforced concrete beams without stirrups?. Composites Part B: Engineering, 107079; 2019.

[17] Olalusi OB. Present state of Eurocode 2 variable strut inclination method for shear design and possible improvement. In: Structures. Elsevier; 2019, Vol. 19, pp. 48-57.

[18] Association Française de Génie Civil. Bétons Fibrés à Ultra-Hautes Performances: Recommandations; Association Française de Génie Civil: Paris, France; 2013 p. 359.

[19] DAfStB. DAfStB-Richtlinie Stahlfaserbeton; DIN: Berlin, Germany; 2012. p. 47.

[20] FIB. Model Code 2010. Final Draft; International Federation for Structural Concrete: Lausanne, Switzerland; 2012. p. 676.

[21] Foster SJ. Design of FRC beams for shear using the VEM and the draft model code approach. fib Bull 2010;57:195-210.

[22] Matthys S, Soetens T. Engineering Model for SFRC Shear Strength Based on MC2010 MCFT Approach. In: High Tech Concrete: Where Technology and Engineering Meet. Cham: Springer; 2018. p. 374-81.

[23] Lee DH, Kim KS, Han SJ, Zhang D, Kim J. Dual potential capacity model for reinforced concrete short and deep beams subjected to shear. Struct Concr 2018;19 (1):76-85.

[24] Colajanni P, Recupero A, Spinella N. Generalisation of shear truss model to the case of SFRC beams with stirrups. Comput Concr 2012;9:227-244. https://doi.org/ 10.12989/ cac.2012.9.3.227.

[25] Spinella N, Colajanni P, Recupero A. Simple plastic model for shear critical SFRC beams. J Struct Eng 2010;136:390-400. https://doi.org/10.1061/(ASCE)ST.1943541X.0000127.

[26] Bernat AM, Spinella N, Recupero A, Cladera A. Mechanical model for the shear strength of steel fiber reinforced concrete (SFRC) beams without stirrups. Mater Struct 2020;53(2):1-20.

[27] Fruchter R, Gluck J, Gold YI. Application of AI programming techniques to the analysis of structures. Comput Struct 1988;30(3):747-53.

[28] Novák D, Lehký D. ANN inverse analysis based on stochastic small-sample training set simulation. Eng Appl Artif Intell 2006;19(7):731-40.

[29] Kawamura K, Miyamoto A, Frangopol DM, Kimura R. Performance evaluation of concrete slabs of existing bridges using neural networks. Eng Struct 2003;25(12): 1455-77.

[30] Papadrakakis M, Lagaros ND. Reliability-based structural optimisation using neural networks and Monte Carlo simulation. Comput Methods Appl Mech Eng 2002;191 (32):3491-507.

[31] Chou JS, Ngo NT, Pham AD. Shear strength prediction in reinforced concrete deep beams using nature-inspired metaheuristic support vector regression. J Comput Civil Eng 2016;30(1):04015002.

[32] Olalusi OB, Spyridis P. Machine learning-based models for the concrete breakout capacity prediction of single anchors in shear. Adv Eng Softw 2020;147:102832.

[33] Tanyildizi H, Cevik A. Modeling mechanical performance of lightweight concrete containing silica fume exposed to high temperature using genetic programming. Constr Build Mater 2010;24(12):2612-8.

[34] Ozbay E, Gesoglu M, Güneyisi E. Empirical modeling ofresh and hardened properties of self-compacting concretes by genetic programming. Constr Build Mater 2008;22(8):1831-1840.

[35] Sarıdemir M. Genetic programming approach for prediction of compressive strength of concretes containing rice husk ash. Constr Build Mater 2010;24(10): 1911-9.

[36] Cabalar AF, Cevik A. Modelling damping ratio and shear modulus of sand-mica mixtures using neural networks. Eng Geol 2009;104:31-40.

[37] Adhikary BB, Mutsuyoshi H. Prediction of shear strength of steel fiber RC beams using neural networks. Constr Build Mater 2006;20:801-11.

[38] Ahn N, Jang H, Park DK. Presumption of shear strength of steel fiber reinforced concrete beam using artificial neural network model. J Appl Polym Sci 2007;103: 2351-8.

[39] Hossain KM, Gladson LR, Anwar MS. Modeling shear strength of medium-to ultrahigh-strength steel fiber-reinforced concrete beams using artificial neural network. Neural Comput Appl 2017;28(1):1119-30.

[40] Naik U, Kute S. Span-to-depth ratio effect on shear strength of steel fiber-reinforced high-strength concrete deep beams using ANN model. Int J Adv Struct Eng 2013;5 (1):29.

[41] Abbas YM, Iqbal Khan M. Influence of fiber properties on shear failure of steel fiber reinforced beams without web reinforcement: ANN modeling. Latin Am J Solids Struct 2016;13(8):1483-98.

[42] Sarveghadi M, Gandomi AH, Bolandi H, Alavi AH. Development of prediction models for shear strength of SFRCB using a machine learning approach. Neural Comput Appl 2019;31(7):2085-94.

[43] Greenough T, Nehdi M. Shear behavior of fiber-reinforced self-consolidating concrete slender beams. ACI Mater J 2008;105(5):468.

[44] Kara IF. Empirical modeling of shear strength of steel fiber reinforced concrete beams by gene expression programming. Neural Comput Appl 2013;23(3-4): 823-34.

[45] Gandomi AH, Alavi AH, Yun GJ. Nonlinear modeling of shear strength of SFRC beams using linear genetic programming. Struct Eng Mech Int J 2011;38:1-25. htt ps://doi.org/10.12989/sem.2011.38.1.001.

[46] Shahnewaz M, Tannert MSAT. Shear strength prediction of steel fiber reinforced concrete beams from genetic programming and its sensitivity analysis. FRC: The Modern Landscape. BEFIB 2016: 9th Rilem International Symposium on Fiber Reinforced Concrete. 2016.

[47] Slater E, Moni M, Alam MS. Predicting the shear strength of steel fiber reinforced concrete beams. Constr Build Mater 2012;26:423-36. https://doi.org/10.1016/j. conbuildmat.2011.06.042 
[48] Islam MS, Alam S. Principal component and multiple regression analysis for steel fiber reinforced concrete (SFRC) beams. Int J Concr Struct Mater 2013;7(4): 303-17.

[49] Islam MS. Simplified shear-strength prediction models for steel-fiber-reinforced concrete beams. In: Proceedings of the Institution of Civil Engineers-Construction Materials; 2018. p. 1-13.

[50] Samui P. Support vector machine applied to settlement of shallow foundations on cohesionless soils. Comput Geotech 2008;35(3):419-27.

[51] Hu B, Su GS, Jiang J, Xiao Y. Gaussian process-based response surface method for slope reliability analysis. Adv Civil Eng; 2019.

[52] Guo M, Hesthaven JS. Reduced order modeling for nonlinear structural analysis using gaussian process regression. Comput Methods Appl Mech Eng 2018;341: 807-26.

[53] Hoang ND, Pham AD, Nguyen QL, Pham QN. Estimating compressive strength of high-performance concrete with Gaussian process regression model. Adv Civil Eng; 2016.

[54] Hensman J, Mills R, Pierce SG, Worden K, Eaton M. Locating acoustic emission sources in complex structures using Gaussian processes. Mech Syst Sig Process 2010;24(1):211-23.

[55] Rasmussen CE. February). Gaussian processes in machine learning. In: Summer School on Machine Learning. Berlin, Heidelberg: Springer; 2003. p. 63-71.

[56] Su G, Jiang J, Yu B, Xiao Y. A Gaussian process-based response surface method for structural reliability analysis. Struct Eng Mech 2015;56(4):549-67.

[57] Su G, Peng L, Hu L. A Gaussian process-based dynamic surrogate model for complex engineering structural reliability analysis. Struct Saf 2017;68:97-109.

[58] Han Q, Gui C, Xu J, Lacidogna G. A generalised method to predict the compressive strength of high-performance concrete by improved random forest algorithm. Constr Build Mater 2019;226:734-42.

[59] Zhang J, Ma G, Huang Y, Aslani F, Nener B. Modelling uniaxial compressive strength of lightweight self-compacting concrete using random forest regression. Constr Build Mater 2019;210:713-9.
[60] Sun Y, Li G, Zhang J, Qian D. Prediction of the strength of rubberized concrete by an evolved random forest model. Adv Civil Eng; 2019.

[61] Cook R, Lapeyre J, Ma H, Kumar A. Prediction of Compressive Strength of Concrete: Critical Comparison of Performance of a Hybrid Machine Learning Model with Standalone Models. J Mater Civ Eng 2019;31(11):04019255.

[62] Thomas J, Ramaswamy A. Mechanical properties of steel fiber-reinforced concrete. J Mater Civ Eng 2007;19(5):385-92.

[63] Melo J. Gaussian processes for regression: a tutorial. Technical Report 2012.

[64] Breiman L. Random forests. Mach Learn 2001;45(1):5-32.

[65] Bertsimas D, Dunn J. Optimal classification trees. Mach Learn 2017;106(7): 1039-82.

[66] Olalusi OB, Viljoen C. Model uncertainties and bias in SHEAR strength predictions of slender stirrup reinforced concrete beams. Struct Concr 2020;21(1):316-32.

[67] Holický M, Retief JV, Sýkora M. Assessment of model uncertainties for structural resistance. Probab Eng Mech 2016;45:188-97.

[68] Olalusi OB, Viljoen C. Assessment of reliability of EN 1992-1-1 variable strut inclination method of shear design provisions for stirrup failure. Struct Concr 2020; 21(1):303-15.

[69] Olalusi OB, Spyridis P. Uncertainty modelling and analysis of the concrete edge breakout resistance of single anchors in shear. Eng Struct 2020 (in review).

[70] EN 1990. (2001). Eurocode - Basis of structural design. European Standard, European Committee for Standardization, Brussels, 2002.

[71] Sýkora M, Holický M, Krejsa J. Model uncertainty for shear resistance of reinforced concrete beams with shear reinforcement according to EN 1992-1-1. Transactions of the VŠB-Technical University of Ostrava, Civil Engineering Series. 2013 Dec 1; 13(2):150-9.

[72] JCSS J. (2001). Probabilistic model code. Joint Committee on Structural Safety. 2001 Mar.

[73] Olalusi OB, Spyridis P. Probabilistic Studies on the Shear Strength of Slender Steel Fiber Reinforced Concrete Structures. Appl Sci 2020;10(19):6955. 